\title{
Examination of the larynx in the histopathology laboratory
}

\author{
L MICHAELS AND RT GREGOR
}

\author{
From the Institute of Laryngology and Otology, University of London, 330 Gray's Inn Road, London \\ WCIX 8EE, UK
}

SUMMARY A method is described for examination of the larynx in the histopathology laboratory. Using a slicing machine, transverse slices of the whole larynx are obtained from which representative histological samples may be prepared. This method offers the advantages of a complete gross examination of the normal and pathological structures of the larynx supplemented by histological studies using any of the methods of paraffin embedding, frozen section, plastic embedding, or electron microscopy on any part of the larynx.

The histological examination of the larynx presents problems that are related to the complex anatomical configuration of that organ. To obtain an adequate picture of the extent of spread of tumour in the laryngectomy specimen multiple sections of the whole specimen are required. This has been achieved by the whole organ serial sectioning method. In this method the whole larynx specimen is cut serially after decalcification and embedding in celloidin ${ }^{1}$ or paraffin wax. ${ }^{2}$ The coronal plane is favoured for the serial sectioning of most laryngeal tumours; epiglottic tumours are sectioned serially in the sagittal plane. $^{3}$

Serial sectioning of the layrnx is, however, far too time-consuming for use in most histopathology laboratories. The method necessitates prior decalcification of the whole organ, a process that requires longer exposure to acid than with smaller blocks of tissue, and therefore leads to inferior histological appearances. By this method also the opportunity for gross study of special areas is lost, and the application of modern histological methods, such as frozen sections, plastic embedding, and electron microscopy is not possible. Whole organ serial sectioning requires long periods of embedding which do not suit the clinical need for a reasonably quick laboratory assessment of the degree of tumour spread.

In a recent 'improved method' of laryngeal examination three large vertical blocks of tissue are taken by sagittal section through tumour and adjacent larynx. ${ }^{4}$ We have used this method extensively and

Received for publication 17 January 1980

$1 *$ found it to be unsatisfactory in certain respects. It does not allow an adequate gross study to be made of the tumour in the larynx, particularly in the case of large or posteriorly situated tumours. The close relationship of the thyroid alae, the cricoid lamina, and the arytenoids in this part of the larynx makes it difficult for well-aligned slices of tissue to be cut by vertical section in this area. The need for whole organ decalcification again results in histologically inferior results. We have tried cutting the strips of larynx for this method on a band-saw before decalcification with resultant improvement of histological appearances but with the separation of mucosa from cartilage due to the method of cutting. Even when decalcified as a whole, the larynx is still tough and elastic, and gross sectioning requires a very sharp knife which does not stay sharp for long. The large, vertically placed slabs of ossified cartilage in proximity to tumour and mucosa make satisfactory microtomy difficult as the softer tissues contract away from the harder ossified cartilage during processing. It is not easy to study intrinsic laryngeal muscles by this method.

The recent introduction of computerised tomography allows a series of horizontal radiographs of the larynx to be taken at $5 \mathrm{~mm}$ intervals. In order to correlate the appearances of such radiographs with pathological changes we have recently turned to horizontal slicing of larynges at similar intervals. We have found a slicing machine to be an ideal means of producing such sections. With this machine a complete gross picture of the tumour in situ in the larynx can be obtained, and very satisfactory histological studies may be carried out in the material so sliced. 


\section{Method}

The larynx is fixed in $10 \%$ buffered formol saline for at least 48 hours. It is then opened by a vertical cut along the midline of the posterior surface, and the lesion is photographed (Fig. 1). After the gross appearances have been described the hyoid bone is carefully dissected off the larynx. If tumour is seen in the pre-epiglottic space, either grossly at this stage or microscopically at a later stage, the hyoid is sectioned transversely by sawing and sampled for histological examination.

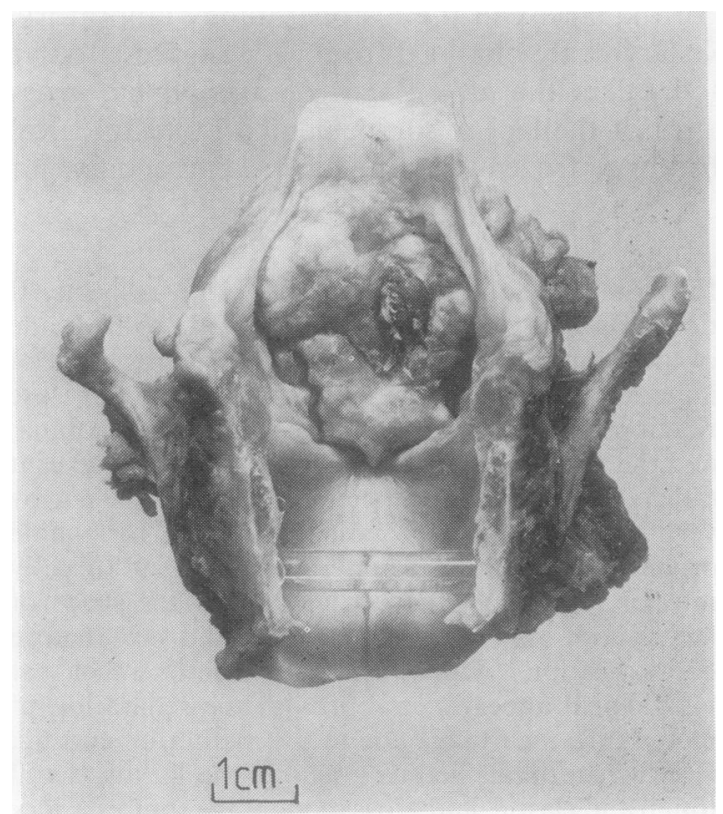

Fig. 1 Gross specimen of carcinoma of larynx. The larynx has been opened by a vertical cut along the midline of the posterior surface and kept open by a glass rod inserted at the lower end. Note the exophytic tumour extending from the epiglottis to the laryngeal ventricles on each side. The right lobe of the thyroid is attached to the specimen and can be seen projecting from the posterior edge of the right thyroid lamina.

The larynx is then sliced transversely in a slicing machine. The machine used by us is an Excel/Boston 10 inch gravity slicing machine.* The machine is supplied with special grindstone equipment for sharpening the circular blade. Slicing is carried out transversely starting at the tip of the epiglottis. The machine is set for cutting slices of $4 \mathrm{~mm}$ thickness by turning the wheel regulating the distance between the

*Supplied by Staines Group (Catering Equipment) Ltd, 15-19 Brewer Street, London W1R 3FL. spherical cutting blade and the safety plate. Four millimetres is the maximum thickness of a block of tissue that can be inserted into a tissue capsule for embedding. The larynx is held in the right hand at its inferior end and wedged firmly against the vertical plate on the movable tray so that its posterior surface is downward. The tip of the epiglottis is exposed for the first slice. The holder supplied with the instrument is not used. Slices are produced by sliding the movable tray sharply against the moving circular blade with the left hand (Fig. 2). When each slice is cut it is carefully orientated so that it represents a

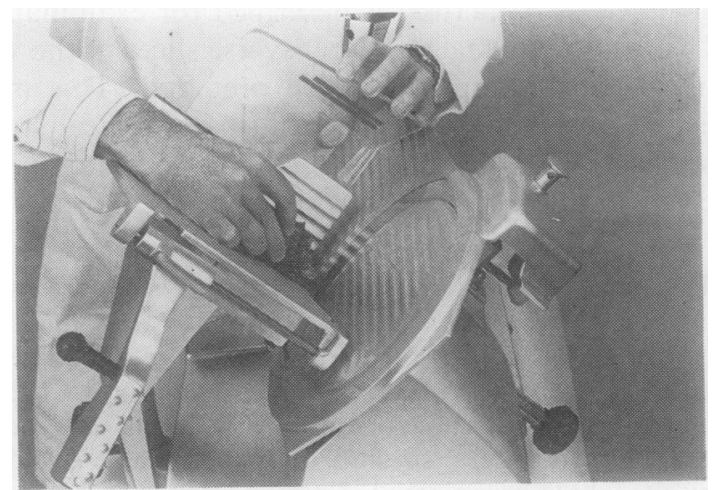

Fig. 2 Slicing the larynx with the slicing machine.

view of the specimen as previously seen from above. A sequence of slices is easily produced in this way, representing the whole larynx by sections which are usually smooth and even (Figs 3 and 4). Occasionally a section from a heavily ossified larynx may become wedged after cutting between the cutting blade and the back metal safety plate, but this may usually be removed intact by widening the space between the cutting blade and the safety plate. The slices of larynx are laid out and identified by a letter in sequence, and each one is photographed by a Polaroid CU5 Land Camera with a 3-inch lens and 1:1 frame using Polaroid Type 107c film. Each slice is examined, and representative blocks are taken for histological study using a sturdy scalpel with a fresh disposable blade. This is usually sufficient to cut through the $4 \mathrm{~mm}$ thick slices of ossified cartilage. The exact position of each block taken for microscopy is marked by drawing corresponding lines with a black felt pen on to the Polaroid photograph. The tissue blocks are carefully orientated by marking the reverse surface to that to be cut after paraffin embedding with India ink. The blocks taken for microscopy are decalcified, processed, and embedded in paraffin wax, and sections are cut at 4 microns and stained in the usual way. Each histological section 

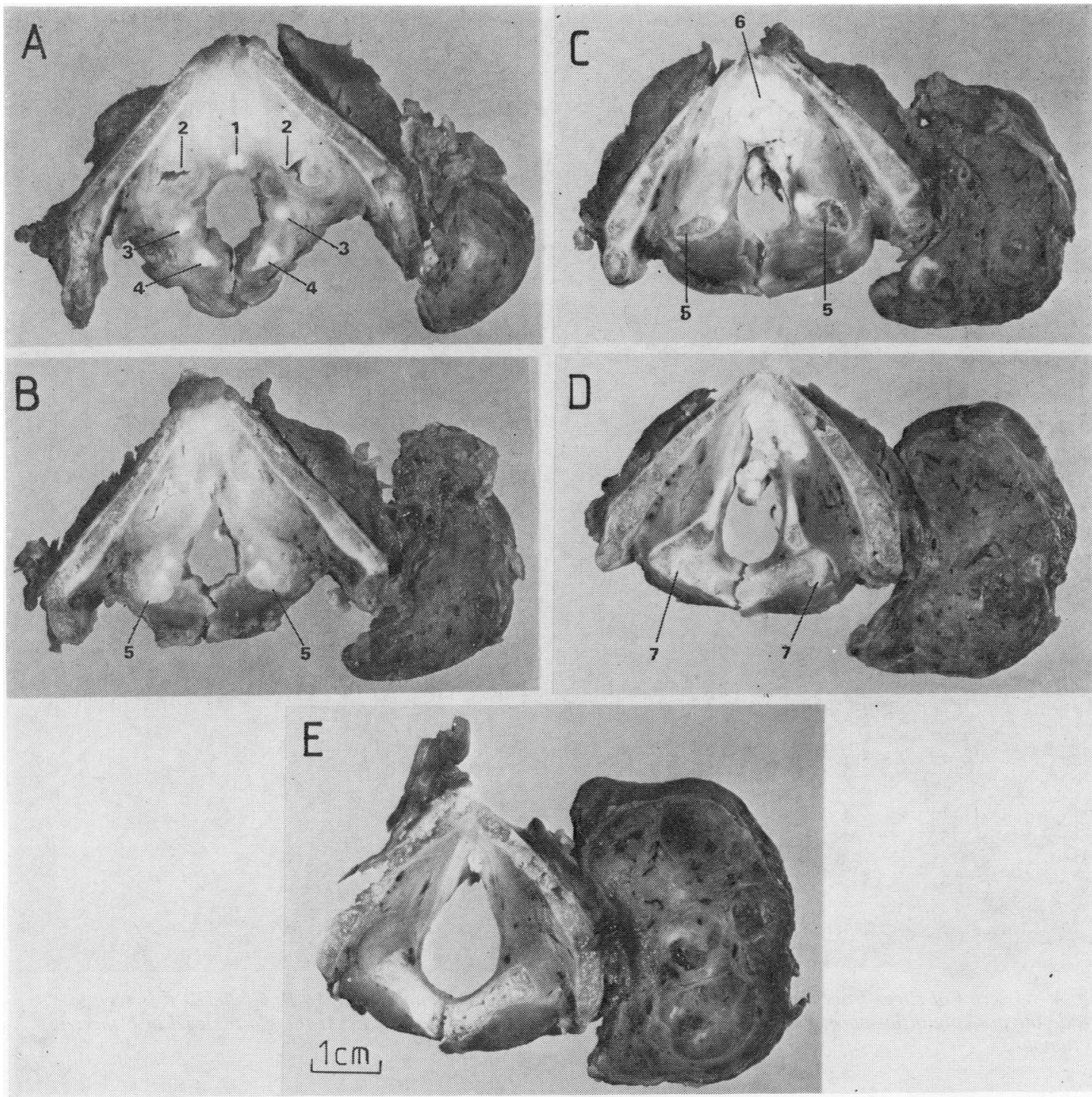

Fig. 3 A series of slices from a larynx with a small right glottic carcinoma. The slices are labelled A to E. The laminae of the thyroid cartilage form a $V$-shaped anterolateral boundary on each slice. The right lobe of the thyroid is present in each slice; it shows nodular colloid changes and in C a calcified nodule. 1 , epiglottic cartilage; 2 , dilated laryngeal saccules; 3, cuneiform cartilage; 4, corniculate cartilage; 5 , arytenoid cartilages, partially ossified; 6, tumour; 7, cricoarytenoid joints.

is easily related to its origin in the original tissue slice by placing the section on to the corresponding Polaroid photograph (which is the same size as the original tissue slice) so that it fits into the shape made by the felt pen lines. Selected areas of tissue may also be subjected to frozen sectioning, plastic embedding, or processing for electron microscopy as required.

\section{Discussion}

By slicing the fixed larynx with a slicing machine, material for an accurate gross study of the larynx is provided quickly and easily (Fig. 5). The following normal structures may be identified in the tissue slices: epiglottis, laminae of the thyroid and cricoid 


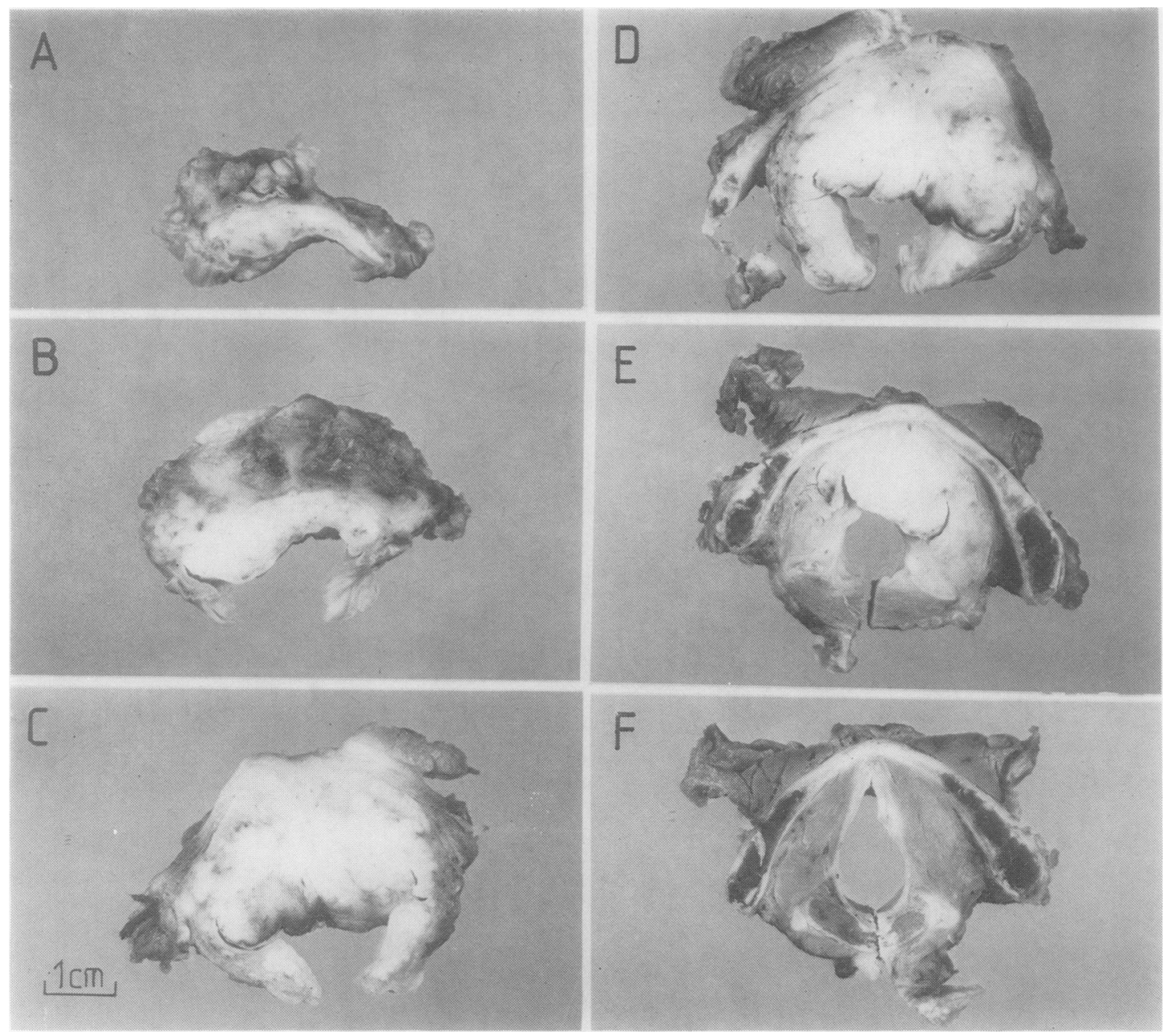

Fig. $4 A$ series of slices from the larynx shown in Fig. 1. There is no tumour in $A$. In B, C, and D it is seen to invade the pre-epiglottic space. Partially ossified arytenoids can be identified at E. At the glottic level in $F$ there is no tumour.

cartilages, corniculate and cuneiform cartilages, ventricular folds (false vocal cords), ventricles and saccules, vocal folds (true vocal cords), inferior horns of the thyroid cartilage, cricoarytenoid joints, cricothyroid joints, and arch of the crioid cartilage. Any small structure that is not displayed on the surface of a block for microscopy may be included in a paraffin block and can be subsequently displayed in histological section by cutting down on to the required area during microtomy. Portions of hypopharynx that are removed with the larynx can be studied in the horizontal sections and the method is particulary suitable for hypopharyngeal carcinoma that has been treated by pharyngolaryngectomy.
In addition to the normal structures mentioned above, the intrinsic laryngeal muscles may be conveniently displayed and sampled for histological examination by this method. One of us (LM) has studied the laryngeal muscles in routine postmortem larynges and in postmortem cases of autonomic failure with multiple system atrophy (Shy-Drager syndrome) in which there was laryngeal muscle abductor muscle palsy. ${ }^{5}$ In this study the intrinsic laryngeal muscles were each separately dissected out and sampled for histology. More recently, postmortem laryngeal intrinsic muscles from a further case of the latter condition have been examined and sampled by slicing the larynx with a slicing machine. 


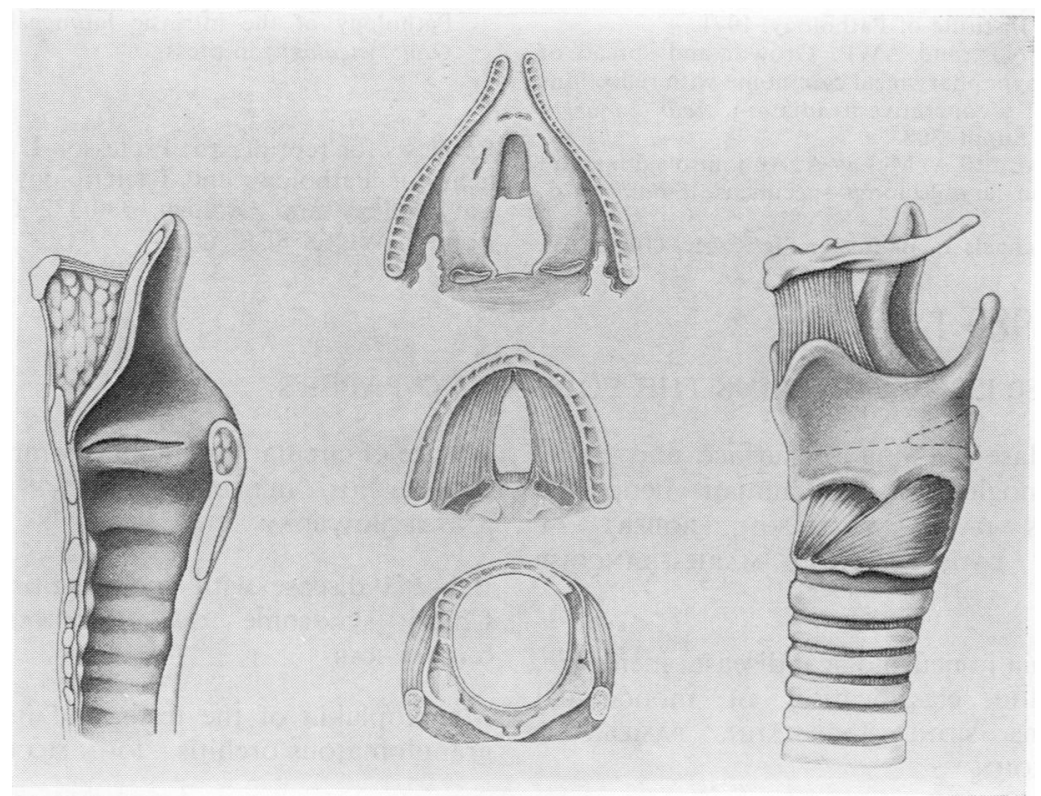

Fig. 5 Diagrams of the larynx. On the right the intact larynx is viewed from the left side. In this drawing the left vocal cord and left arytenoid cartilage are shown on dotted lines. On the left the interior of the right half of the larynx is observed after removing the left half. Note the extensive pre-epiglottic space in the latter drawing anterior to the epiglottis.

Typical transverse sections of the larynx are depicted in the three central drawings. The upper one is taken near the base of the epiglottis. Note the epiglottic cartilage, the two saccules, and the corniculate cartilages posteriorly. The pyriform fossa of the hypopharynx invaginates just internal to the thyroid cartilage on each side. The middle drawing is at the level of the true vocal cords. Note the arytenoid cartilages and cricoarytenoid joints posterior to each cord and the thyro-arytenoid muscle lateral to it. The lowest transverse section drawing is shown at the level of the cricoid ring and thyroid cornua. The lateral muscles are the cricothyroids and the posterior ones are the posterior cricoarytenoids.

The individual intrinsic muscles were easily recognised in the transverse sections so produced. The method proved especially valuable in providing serially cut slices of the atrophic posterior cricoarytenoid, a specific feature of autonomic failure with multiple system atrophy.

Larynges with neoplastic growth can be satisfactorily studied by this method and an accurate picture built up based on gross and microscopic examination of the relationship of the tumour to normal structures. This is a far less laborious procedure than the serial section cutting method. The tissue blocks taken for histology need little decalcification time as they are small so that the stained sections show minimal harmful acid-produced effects. Mucosa seems to adhere to cartilage better by such transverse cutting. Blocks may be taken from the transverse slices for a wide variety of modern histological, histochemical, and electron microscopical procedures. A report may be issued on the specimen within eight days of the laryngectomy operation. The $4 \mathrm{~mm}$ slices used in this method provide a thickness close to the $5 \mathrm{~mm}$ section obtained with computerised tomography. We are at present using this method to help in the interpretation of the computerised tomography scans.

We are indebted to Professor DFN Harrison for suggesting the use of the slicing machine in the examination of the larynx and for his encouragement and advice in the development of the method described.

\section{References}

1 Tucker GF. A histological method for the study of the spread of carcinoma within the larynx. Ann Otol 1961; 70:910-21.

${ }^{2}$ Tucker GF. Coronal Section Atlas-Normal Larynx. 
Armed Forces Institute of Pathology. 1971.

${ }^{3}$ Olofson J, Van Nostrand AWP. Growth and spread of laryngeal and hypopharyngeal carcinoma with reflections on the effect of preoperative it radiation. Acta Otolaryngologica 1973; Suppl. 308.

4 Browning GG, Busuttil A, McLay A. An improved method of reporting on laryngectomy specimens. J Path 1976; 119:101-4.

${ }^{5}$ Guindi GM, Michaels L, Bannister Sir Roger, Gibson W.
Pathology of the intrinsic laryngeal muscles. Clinical Otolaryngology; in press.

Requests for reprints to: Professor L Michaels, Department of Pathology and Bacteriology, The Institute of Laryngology and Otology, 330/332 Gray's Inn Road, London WC1X 8EE, UK.

\section{The July 1980 Issue}

\section{THE JULY 1980 ISSUE CONTAINS THE FOLLOWING PAPERS}

Immunoperoxidase staining of surface and intracellular immunoglobulin in human neoplastic lymphoid cells DAVID Y MASON, ROBERT CF LEONARD, GUY LAURENT, AND MARIE-FRANÇOISE GOURDIN

Paraproteinaemia in neurological disease: incidence, associations, and classification of monoclonal immunoglobulins SIDNEY N KAHN, PAMELA G RICHES, AND J KOHN

Platelet hyperactivity in sickle-cell disease: a consequence of hyposplenism MW KENNY, AJ GEORGE, AND J STUART

Liver function and the diagnostic significance of biochemical changes in the blood of African children with sickle cell disease UP ISICHEI

Intermittent heparin treatment does not induce hypercoagulability in haemodialysed patients $F$ PUSINERI, A BINI, L MUSSONI, G REMUZZI, AND MB DONATI

Enzyme-linked immunosorbent assay for quantitation of toxoplasma antibodies in human sera $\mathbf{A M}$ VAN LOON AND J VAN DER VEEN

ELISA for toxoplasma antibody detection: a comparison with other serodiagnostic tests A BALSARI, G POLI, V MOLINA, M DOVIS, E PETRUZZELLI, A BONIOLO, AND E ROLLERI

An evaluation of the ToxHA test for the detection of antibodies to Toxoplasma gondii in human serum ALAN H BALFOUR, JOHN B BRIDGES, AND JOHN P HARFORD

Antibody to synthetic poly dAT: correlation with antibody to native DNA and specificity for SLE PAUL DAVIS AND DREW MAKINEN
Nature of circulating immune complexes in infective endocarditis JILL BURTON-KEE, P MORGAN-CAPNER, AND JF MOWBRAY

Behçet's disease with endocarditis and the BuddChiari syndrome GSA MCDONALD AND JANET GAD-AL-RAB

Malakoplakia of the testis and its relationship to granulomatous orchitis JOHN MCCLURE

Hydrocolpos with peritonitis in the newborn I GUPTA AND AJ BARSON

Lysosomal naphthylamidase activity as a possible aid in cytological screening JACQUELINE A MILLETT, YVONNE CHIN, LUCILLE BITENSKY, J CHAYEN, AND OAN HUSAIN

Comparison of cytological 'jet-wash' specimens and histology in endometrial carcinoma ELSA SEGADAL, OLE ERIK IVERSEN, AND MAGNAR ULSTEIN

Use of the Streptosec test for grouping betahaemolytic streptococci PW ROSS, A NICOLL, AND CG CUMMING

Dipslide cultures in the investigation of suprapubic urinary bladder aspirates of infants and children ANJA AI KOSTIALA AND J PYLKKÄNEN

\section{Technical methods}

Examination of skin window preparations by transmission electron microscopy RJ SOKOL, PD NORRIS, AND G HUDSON

A simple method for recording thin-layer acrylamide gel electropherograms U HLA-PE AND U TIN-WIN

Letters to the Editor

Book reviews

Copies are still available and may be obtained from the PUBLISHING MANAGER, BRITISH MEDICAL ASSOCIATION, TAVISTOCK SQUARE, LONDON WC1H 9JR, price $£ 3.00$, including postage 